\title{
A Time-Frequency Research for Ultrasonic Guided Wave Generated from the Debonding Based on a Novel Time-Frequency Analysis Technique
}

\author{
Junhua Wu, Zheshu Ma, and Yonghui Zhang \\ School of Automotive and Traffic Engineering, Nanjing Forestry University, Nanjing, Jiangsu 210037, China \\ Correspondence should be addressed to Junhua Wu; bjtime13@126.com
}

Received 18 September 2016; Accepted 9 January 2017; Published 9 February 2017

Academic Editor: Brij N. Agrawal

Copyright @ 2017 Junhua Wu et al. This is an open access article distributed under the Creative Commons Attribution License, which permits unrestricted use, distribution, and reproduction in any medium, provided the original work is properly cited.

Carbon fibre composites have a promising application in the future of the vehicle, because of their high strength and light weight. Debonding is a major defect of the carbon fibre composite. The time-frequency analysis is fundamental to identify the defect on ultrasonic nondestructive evaluation and testing. In order to obtain the instantaneous frequency and the peak time of modes of the ultrasonic guided wave, an algorithm based on the Smoothed Pseudo Wigner-Ville distribution and the peak-track algorithm is presented. In the algorithm, a masking step is proposed, which can guarantee that the peak-track algorithm can automatically exact the instantaneous frequency and the instantaneous amplitude of different modes on the Smoothed Pseudo Wigner-Ville distribution. An experiment for detecting the debonding for a type of carbon fibre composite is done. The presented algorithm is employed on the experimental signals. The processed result of experimental signals reveals that the defect can stimulate new modes, and there is a quantitative relationship between the defect size and the frequency of the new mode. The presented technique provides a valuable way to detect the presentence, calculate the size, and locate the position of the debonding defect.

\section{Introduction}

Carbon fibre composite is a type of composite material with a high strength. The specific strength and specific stiffness are better than other lightweight materials. Therefore, it is widely employed in aerospace domain and military products. The material can obviously reduce the weight of automobile. The material has been applied on construction elements and cover parts of transmission system of chassis and automobile bodies. Debonding defect is a major problem of the carbon fibre composites. A great number of scholars have done investigations of the nondestructive evaluation and testing (NDE/NDT) for the defect [1-4].

At present, ultrasonic guided wave (GW) testing has emerged as a very prominent active scheme. It offers an effective method to estimate the location, severity, and type of defects and has been used on NDE/NDT of the carbon fibre composites $[2,5,6]$. Separating different modes of the GW and obtaining the corresponding time-frequency distributions (TFD) are vital for the inspection of the defect.
A number of time-frequency analysis techniques have been explored for analysis of GW signals. Prosser et al. [7] used a pseudo Wigner-Ville distribution to process GW signals for material characterization of composites. Kercel et al. [8] used Bayesian parameter estimates to separate multiple modes in GW signals obtained from laser ultrasonic on a workpiece manufacturing assembly line. Mahadev et al. [9] employed the short time Fourier transform (STFT) to extract a suitable parameter for a tomographic image reconstruction mapping structural defects. Osegueda et al. [10] used HilbertHuang transform (HHT) to process GW signals in plate structures. This technique allows for separating of the GW signal into intrinsic mode functions (IMF) and a residue. Hilbert transform is done for IMFs to determine the energy time signal of each mode, enabling the easy location and characterization of the notch. The wavelet analysis is widely used [11-17]; a good many works were done about applications of wavelet transform (WT) for the GW signals. Dhital and Lee [13] used WT for detection of cracks, which was also realized. Paget et al. [14] proposed a new damage-detection technique 
based on WT with a new basis. Y. Y. Kim and E.-H. Kim evaluated the effectiveness of WT analysis for studying the wave dispersion [16].

The instantaneous frequency (IF) and the peak time of the instantaneous amplitude (IA) are significant parameters of the GW mode. Tracking the IF change may provide way to identify the presence and the size of the defect. Peak time can evaluate the distance between the defect and the probe; on this basic, we can locate the defect. HHT can realize these operations. But the frequency resolution is a limitation. Ref. [18] reveals that when the ratio between a relatively low frequency and a relatively high frequency becomes close to 0.75 (low frequency/high frequency) two components of signals cannot be separated.

Although Ensemble Empirical Mode Decomposition (EEMD) can overcome the mode mixing in certain degree, this limitation still exists in EEMD. Wigner-Ville distribution has a fine time-frequency resolution and can reach the low boundary of Heisenberg uncertainty principle. And Smoothed Pseudo Wigner-Ville distribution (SPWVD) can remove the cross-term in frequency direction and time direction of the time-frequency panel. The peak-track algorithm can extract the IF and IA of a monocomponent signal. For multicomponent signals, mashing operation should be conducted to guarantee that just one mode in time-frequency panel is processed by using the peak-track algorithm. To realize automatically mashing operation, a step based on the minimal and maximal values of a statistic parameter of the time-frequency distribution is presented to extract and separate valuable modes on the time-frequency panel. Combining with the peak-track algorithm and SPWVD, an algorithm that can compute the IF and IA of the GW signal is proposed. After that, a time-frequency analysis of debonding of a type of carbon fibre composites is conducted to discuss the feasibility of identifying the presence, size, and location of the defect.

The rest of this paper is organized as follows. Section 2 illustrates the principle of algorithm. Section 3 provides an illustration of the presented method and a contrast between it and several traditional time-frequency analysis techniques. Section 4 describes the detail of the experiment. Section 5 shows an analysis of the time-frequency analysis of the experimental signals employing the presented technique. Finally, Section 6 concludes the paper.

\section{Principle of the Algorithm}

The processing steps of the extension algorithm are shown in Figure 1 and are as follows.

(1) Smoothed Pseudo Wigner-Ville distribution: WignerVille distribution has a fine time-frequency resolution and can reach the low boundary of Heisenberg uncertainty principle. It is defined as follows [19]:

$$
\operatorname{WVD}_{s}(t, f)=\int_{-\infty}^{\infty} s\left(t+\frac{\tau}{2}\right) s\left(t-\frac{\tau}{2}\right) e^{-j 2 \pi f \tau} d \tau
$$

SPWVD can suppress the cross-term in frequency and time directions. To get time-frequency panel with a promising time-frequency resolution, we employ SPWVD on testing signals. And the formula of SPWVD is as follows [20]:

$$
\begin{aligned}
\mathrm{SPW}_{s}(n, \Theta) & =\sum_{k=-L+1}^{L-1}|h(k)| \\
\cdot & \sum_{l=M+1}^{M-1} g(l) s(n+l+k) s^{*}(n+l-k) e^{-j 2 k \Theta},
\end{aligned}
$$

where $g(l)$ and $h(k)$ are smoothing window functions in time direction and frequency direction, respectively. $s$ is an analyzed signal and $n$ and $\Theta$ are time variable and frequency variable, respectively.

(2) Extracting and separating valuable areas of the timefrequency panel. As mentioned above, significant timefrequency coefficients tend to cluster in groups or regions. To extract and separate them, we do following steps: (1) sum coefficients of each frequency-indexed group, and the calculation formula is

$$
E(\Theta)=\sum_{n=1}^{N} \operatorname{SPW}_{s}(n, \Theta),
$$

where $N$ denotes the number of the time variable of the timefrequency panel. (2) Calculate minimal values and maximum values of summations. Lines of the minimal values in the time-frequency panel can separate the valuable areas. And the maximum value evaluates whether the energy of a mode is strong enough to be kept. (3) Conduct threshold processing for the maximum values. And the equation is defined as follows:

$$
E(\Theta)_{\max }= \begin{cases}\text { keep } & E(\Theta)_{\max }>T_{0} \\ \text { discard } & E(\Theta)_{\max } \leq T_{0},\end{cases}
$$

where $E(\Theta)_{\max }$ means the maximum of $E(\Theta) . T_{0}$ denote the threshold for sift valuable modes. A mode with a strong energy is more valuable for detecting the debonding defect, so a threshold process is introduced to improve calculation efficiency. Moreover, the noise can also be removed by this operation. After this step, the areas of the timefrequency panel corresponding to the significant modes can be extracted and separated.

(3) Peak-track algorithm. Conduct the peak-track algorithm for the significant areas of SPWVD to obtain the primary IF and the primary IA of different models. The principle of the peak-track algorithm can be found in [21]. And then, the IFs and IAs of significant models can be obtained, which provides detailed information of the time-frequency distribution of the GWs for detecting defects. On the basis of these information, we can discuss the debonding of testing objects.

(4) Threshold process for the primary IF and the primary IA: to suppress the influence of noise, a threshold process for the primary IA is conducted. The equation is defined as

$$
A= \begin{cases}A_{0} & A_{0}>T_{A} \\ 0 & A_{0} \leq T_{A}\end{cases}
$$


where $A$ denote the final IAs of modes and $A_{0}$ denote the primary IAs of modes. $T_{A}$ is a threshold to suppress the influence of noise. Correspondingly, the primary frequency is where the final IA is set to 0 . And then, we can research the peak time $t_{\text {peak }}$ of the final IA as follows:

$$
t_{\text {peak }}=\arg \max \{\operatorname{IA}(t)\}
$$

In Section 3, the detail of the present algorithm will be illustrated with a sample signal.

\section{Illustration of the Presented Algorithm}

We construct a sample signal to illustrate the presented algorithm as follows:

$$
\begin{aligned}
& s^{s}(t)= \\
& s_{1} s_{1}(t)+c_{2} s_{2}(t)+c_{3} s_{3}(t), \\
& s_{1}(t)= \begin{cases}0, & 0 \leq t \leq 0.0036 \\
\left(\frac{1250}{3}\right)(t-0.0036) \sin (75000 \times 2 \pi t), & 0.0036<t \leq 0.006 \\
-\left(\frac{2500}{9}\right)(t-0.006) \sin (75000 \times 2 \pi t), & 0.006<t \leq 0.0096 \\
0, & 0.0096<t \leq 0.012,\end{cases} \\
& s_{2}(t)= \begin{cases}0, & 0 \leq t \leq 0.0024 \\
\left(\frac{1250}{3}\right)(t-0.0024) \sin (100000 \times 2 \pi t), & 0.0024<t \leq 0.0048 \\
-\left(\frac{2500}{9}\right)(t-0.0048) \sin (100000 \times 2 \pi t), & 0.0048<t \leq 0.0084 \\
0, & 0.0084<t \leq 0.012,\end{cases} \\
& s_{3}(t)= \begin{cases}0, & 0 \leq t \leq 0.0012 \\
\left(\frac{1250}{3}\right)(t-0.0012) \sin (200000 \times 2 \pi t), & 0.0012<t \leq 0.0036 \\
-\left(\frac{2500}{9}\right)(t-0.0036) \sin (200000 \times 2 \pi t), & 0.0036<t \leq 0.0072 \\
0, & 0.0072<t \leq 0.012 .\end{cases}
\end{aligned}
$$

The sampling frequency is $150 \mathrm{kHz}$. A noise with a strength of 0.1 is added to the signal to test the suitability of the algorithm. The sample signal contains three frequencies at 75, 100, and $200 \mathrm{kHz}$. In (7), $c_{1}$ and $c_{2}$ are 1 , and $c_{3}$ is 0.4 . The curve of the sample signal in time domain is shown in Figure 2.

(1) Smoothed Pseudo Wigner-Ville Distribution. We employ SPWVD for the sample signal to obtain the corresponding time-frequency panel, which is shown in Figure 3. A contrast among SPWVD, STFT, and EEMD is done. STFTs representation with long and short windows of the sample signal is shown in Figures 4 and 5. The STFT representation can be obtained by [22]

$$
\operatorname{STFT}_{s}(t, f)=\int_{-\infty}^{\infty} s(\tau) h^{*}(\tau-t) e^{-j 2 \pi f \tau} d \tau,
$$

where $h^{*}(\tau-t)$ is the conjugate function of window function $h(\tau-t)$. It is different to keep a high frequency resolution and a high time resolution at the same time for time-frequency representation techniques, which is illustrated in Figures 4 and 5. When the window is long, the frequency resolution is good at the cost of the time resolution, as shown in Figure 4.
By contrast, as can be seen in Figure 5, when the window is short, the time resolution is good, but the modes at 75 and $100 \mathrm{kHz}$ cannot be separated.

Wigner-Ville distribution has a fine time-frequency resolution and can reach the low boundary of Heisenberg uncertainty principle. However, for multicomponent signals, it suffers from an inevitable cross-term interference. SPWVD can remove it in frequency direction and time direction of the time-frequency panel. As can be seen in Figure 3, the time resolution and frequency resolution of the time-frequency are promising. Moreover, no interference is in the representation.

In EEMD, signals are decomposed as

$$
s(t)=\sum_{i=1}^{n} c_{i}(t)+r_{n}(t)
$$

where $c_{i}(t)$ is the $i$ th IMF and $r_{n}(t)$ is the residual signal which represents the slowly varying or constant trend of the signal. According to [23], an IMF with a high coefficient of correlation between it and the analyzed signal means that this IMF is an important component. Figure 6 presents the coefficients of correlation between different IMFs and 


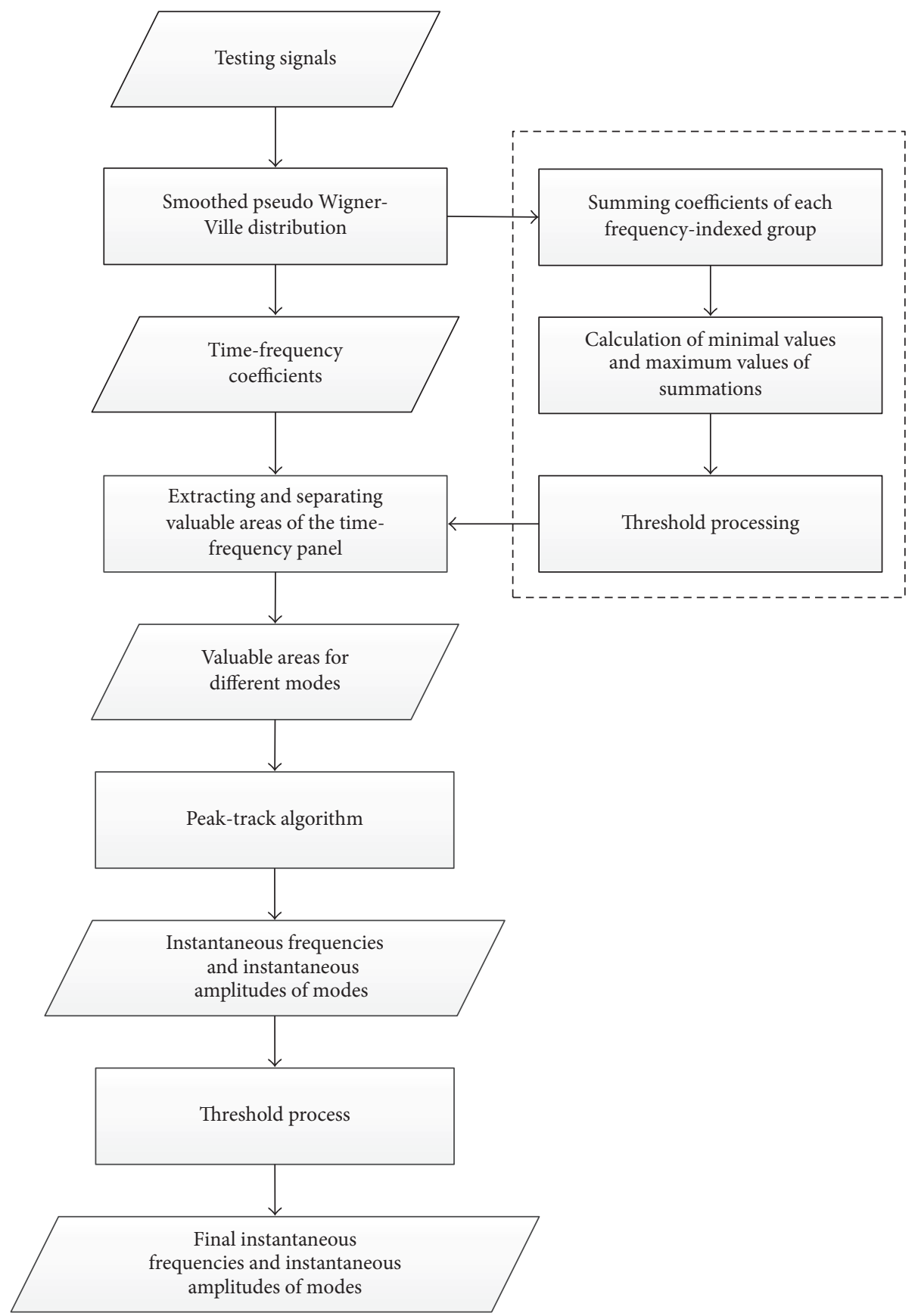

FIGURE 1: The different steps of the algorithm presented in the paper.

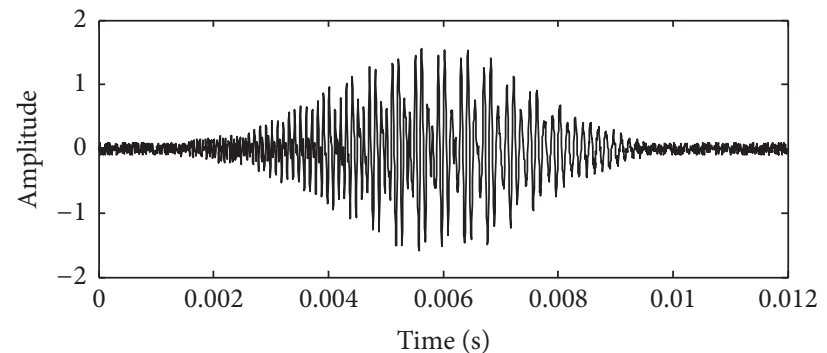

Figure 2: The curve of the sample signal in time domain. 


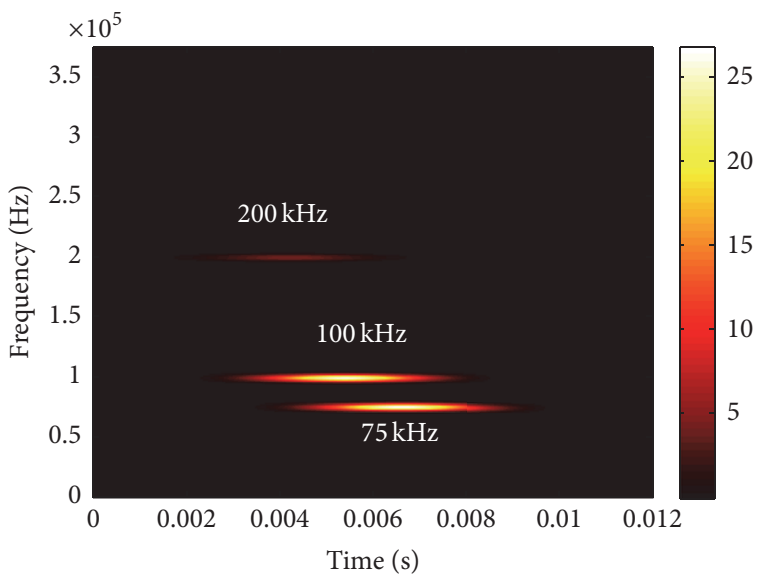

(a)

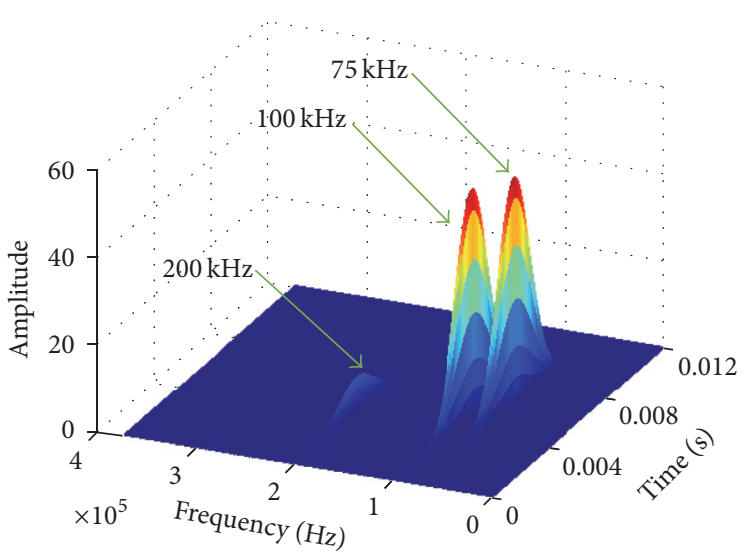

(b)

FIGURE 3: The SPWVD representation of the sample signal: (a) two-dimensional figure and (b) three-dimensional figure.

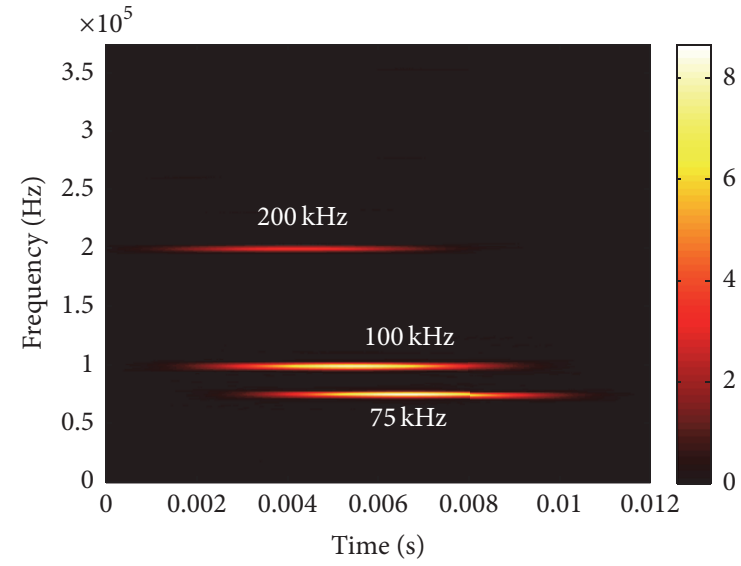

(a)

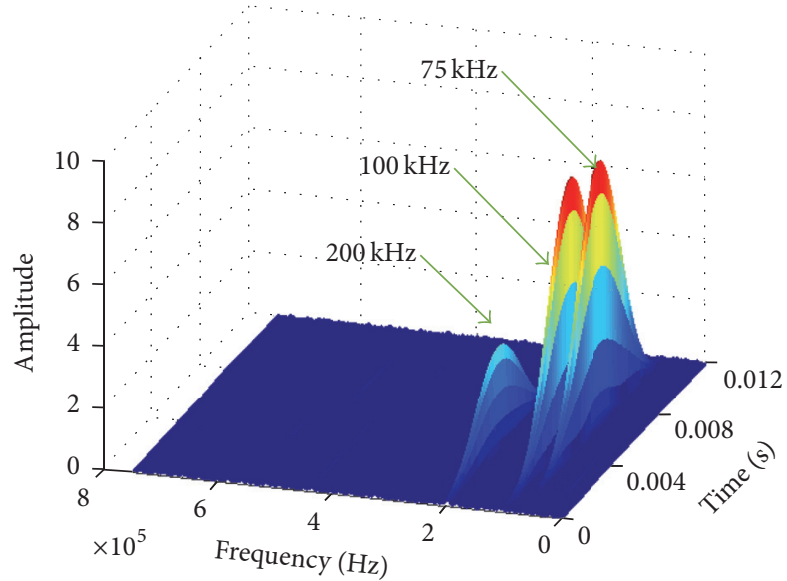

(b)

FIGURE 4: The STFT representation with a long window of the sample signal: (a) two-dimensional figure and (b) three-dimensional figure.

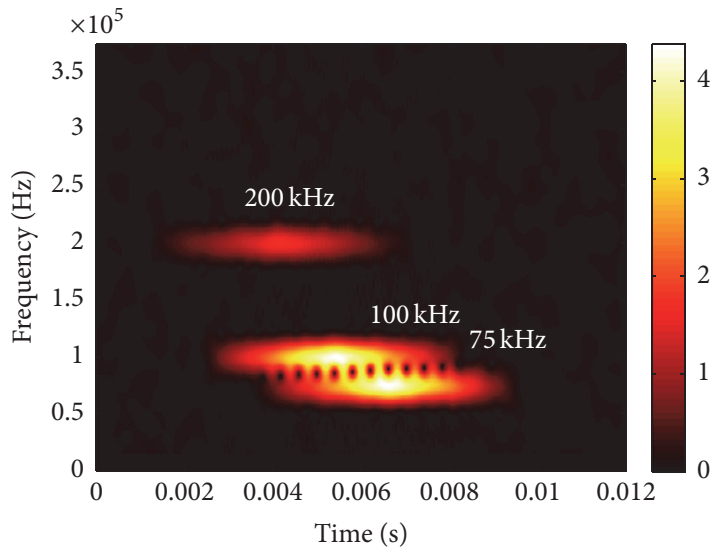

(a)

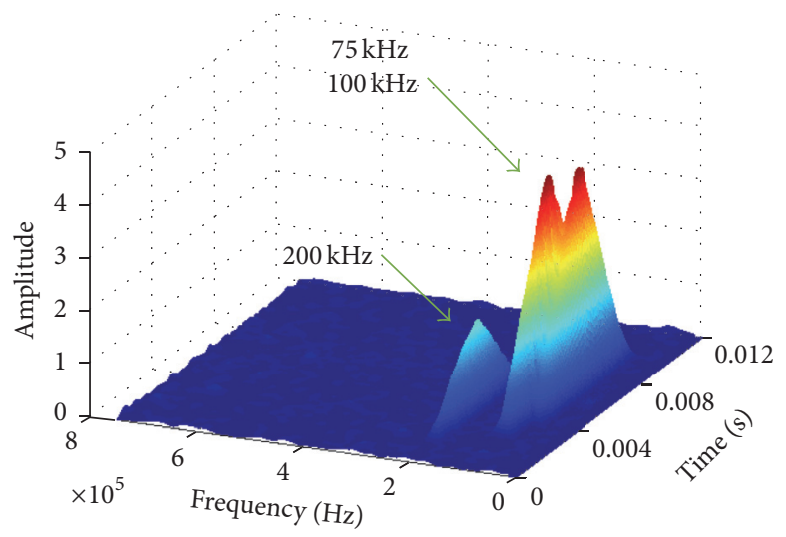

(b)

FiguRE 5: The STFT representation with a short window of the sample signal: (a) two-dimensional figure and (b) three-dimensional figure. 


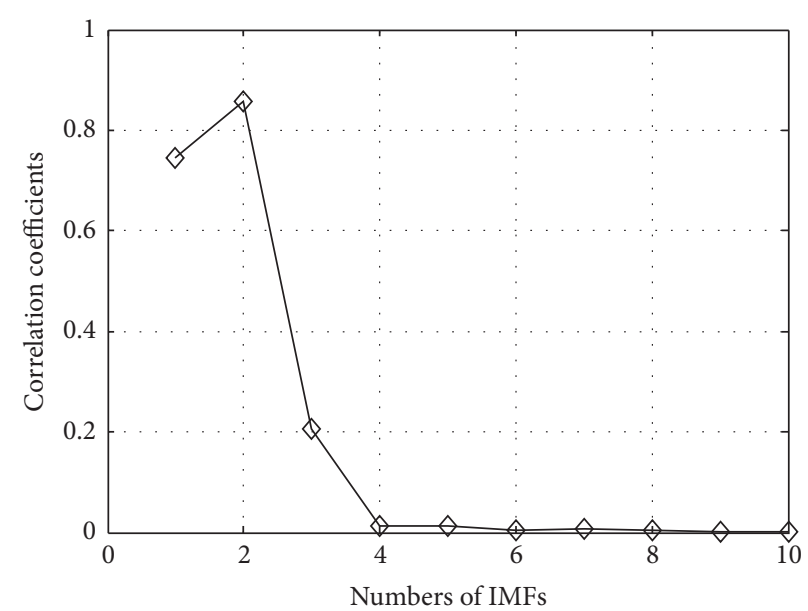

FIGURE 6: Correlation coefficients between each IMF and the sample signal.
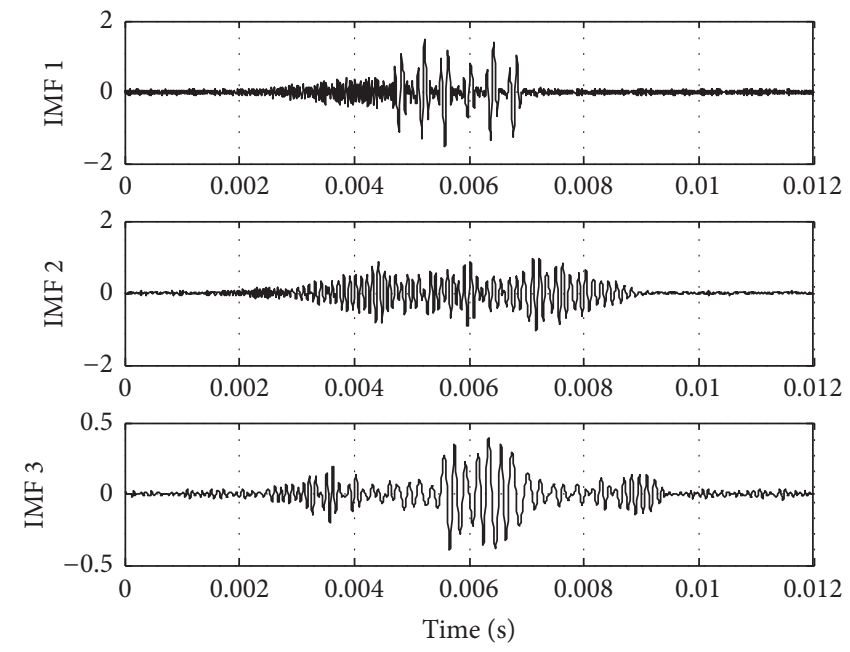

FIgURE 7: The IMFs 1-3 in the EEMD of the sample signal.

the sample signal. We can learn that IMFs $1-3$ are vital components of the signal, as the coefficients of correlation are relatively greater. So these IMFs are shown in Figure 7. It is visible that the mode mixing occurs in EEMD. Ref. [18] reveals that, when the ratio between a relatively low frequency and a relatively high frequency becomes close to 0.75 (low frequency/high frequency), two components of signals cannot be separated. Therefore, the poor frequency resolution is a limitation of EEMD for its application.

(2) Extracting and Separating Valuable Areas of the TimeFrequency Panel. As mentioned above, peak-track algorithm can obtain the peak values and the corresponding coordinates in different time points of the time-frequency panel. However, when more than one mode exist on the panel, some errors may occur. To resolve it, we should extract and separate different modes from the time-frequency representation. At first, we sum coefficients of each frequency-indexed group by using (3). And the different frequency-group summations of the time-frequency panel of the sample signal are illustrated in Figure 8.

Afterward, we calculate minimal values and maximum values of summations. In the calculation process of the sample signal, 139 minimal values are obtained, which means that 138 modes will be extracted. According to (7), the sample signal includes 3 modes. The error root is from the noise. To remove the effectiveness of noises, we employ (4) to sift the valuable mode and set the threshold to 100 in (4). Three groups of minimal values are kept, meaning three modes are kept.

Finally, we filter the SPWVD representation with the kept groups of minimal values. Figure 9 shows the filtering result. As shown in Figure 9, three modes are extracted and separated.

(3) Peak-Track Algorithm. We employ the peak-track algorithm on the filtering result of the SPWVD of the sample signal shown in Figure 9. The primary IAs and IFs are 


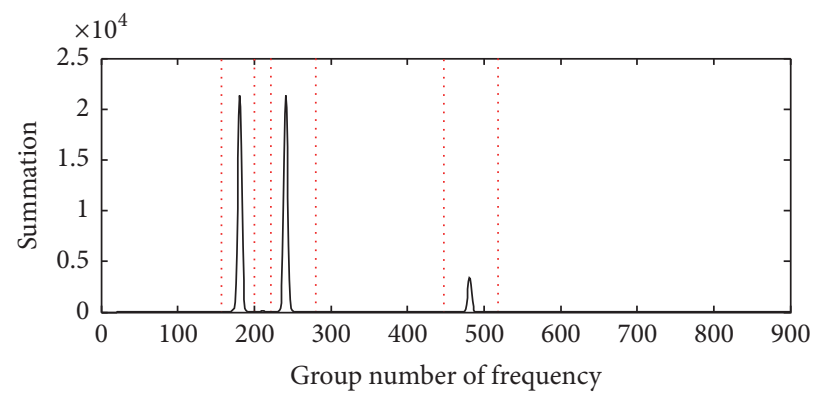

FIGURE 8: The different frequency-group summations of the time-frequency panel of the sample signal.

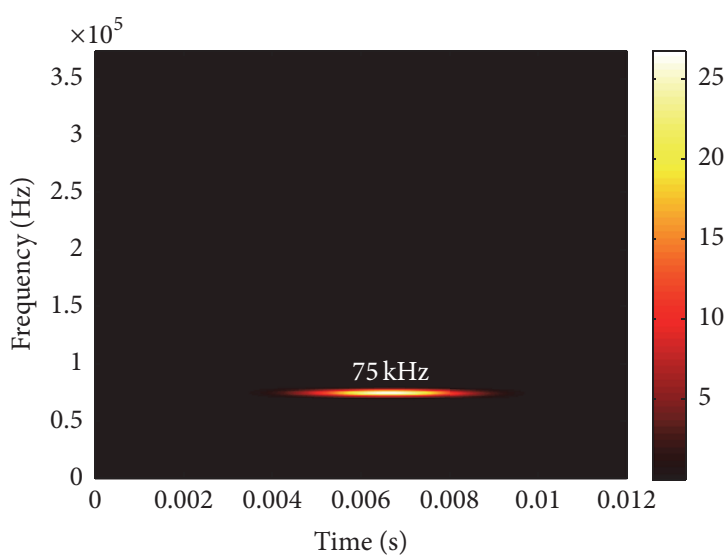

(a)

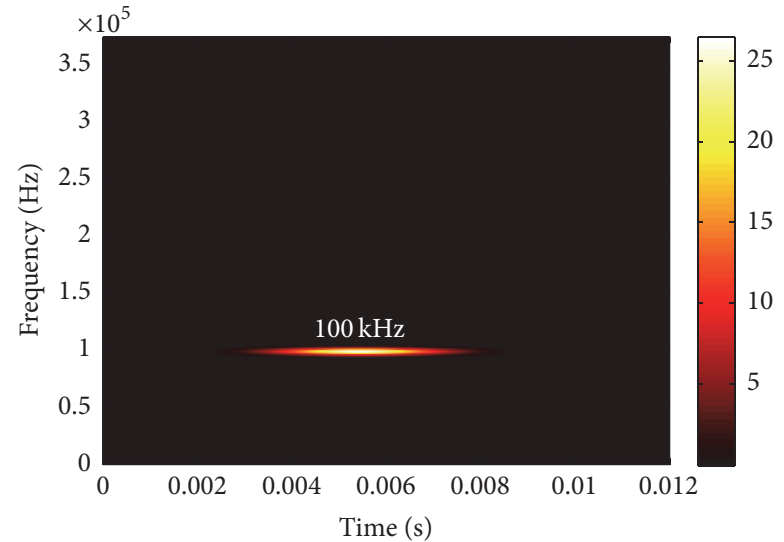

(b)

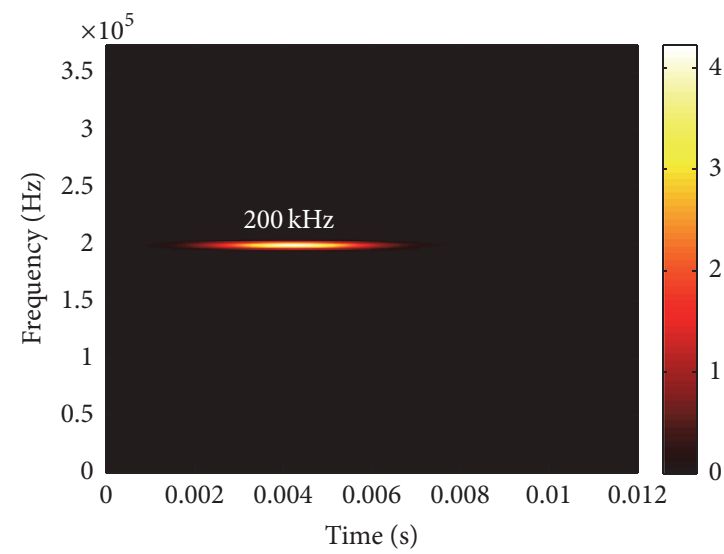

(c)

FIGURE 9: The filtering result of the SPWVD of the sample signal.

obtained and are shown in Figure 10. Because of noise, calculation errors occur in IFs.

(4) Threshold Process for the Primary IF. To suppress the influence of the noise, (5) is applied on the IA. The threshold $T_{A}$ is set to 0.1. The final IFs are got, as shown in Figure 11. As can be seen in Figure 11, the IFs of the three mode are 75,100 , and $200 \mathrm{kHz}$, respectively. The calculations of IFs are in a high accuracy. Moreover, the time resolution is also in a promising accuracy. For example, the peak times of the different modes are $0.004201 \mathrm{~s}, 0.005402 \mathrm{~s}$, and $0.00006597 \mathrm{~s}$, and the calculation errors are $0.00001 \mathrm{~s}, 0.00002 \mathrm{~s}$, and $0.00003 \mathrm{~s}$. These results prove the validity of the presented algorithm.

\section{Detail of the Experimental}

The material of the specimen is T300/QY8911 composite materials. The size is $500 \mathrm{~mm} \times 300 \mathrm{~mm} \times 2 \mathrm{~mm}$ and contains 10 layers. The physical parameters of the material are as follows: $E_{1}=135 \mathrm{GPa}, E_{2}=8.8 \mathrm{GPa}, E_{3}=8.8 \mathrm{GPa}, G_{12}=$ $4.47 \mathrm{GPa}, G_{23}=3.45 \mathrm{GPa}, G_{13}=4.47 \mathrm{GPa}, \nu_{12}=0.3$, $v_{23}=0.34, v_{13}=0.3$, and $\rho=1560 \mathrm{~kg} / \mathrm{m}^{3}$. The photograph 


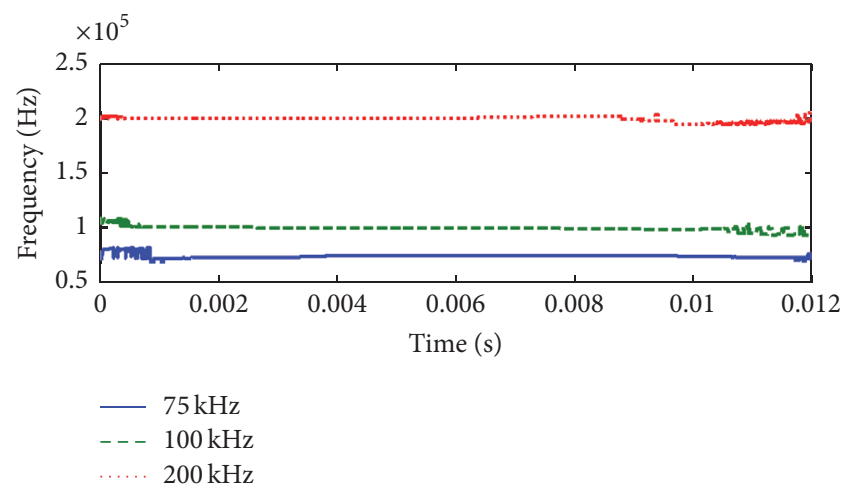

(a)

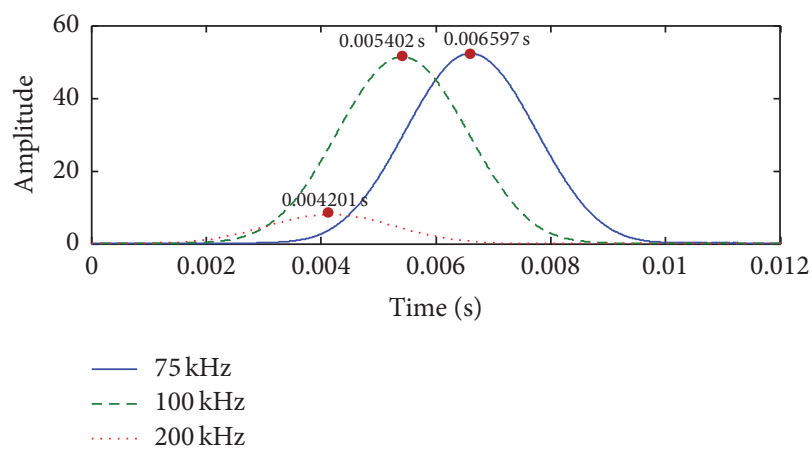

(b)

FIGURE 10: The primary IFs and IAs obtained by using the peak-track algorithm: (a) IF and (b) IA.

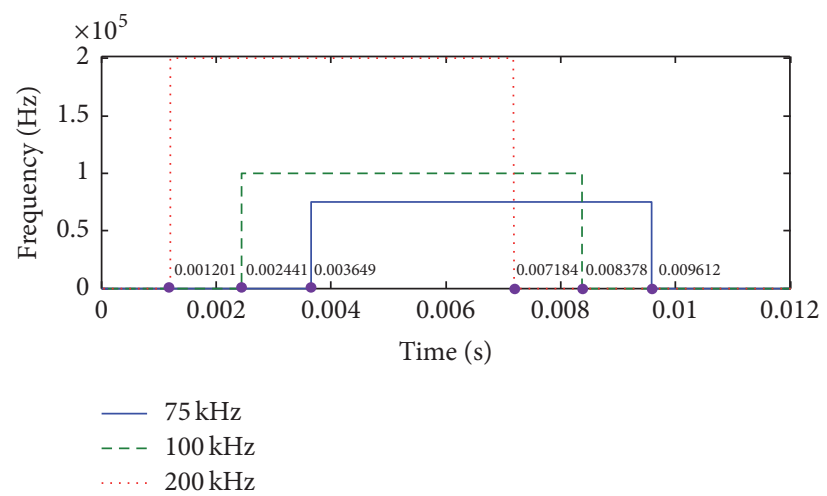

FIGURE 11: The final IFs of the sample signal by using the presented algorithm.

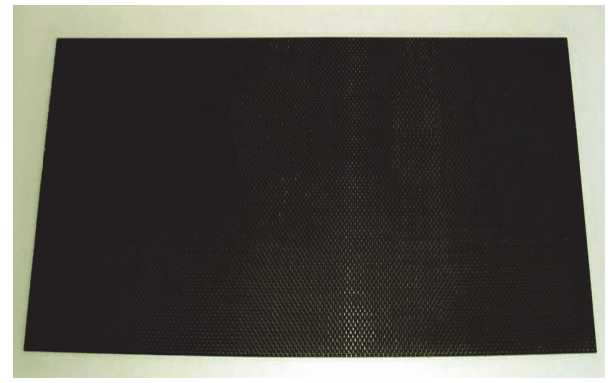

Figure 12: The photograph of the specimen.

of the experimental specimen is shown in Figure 12. The corresponding size diagram is presented in Figure 13. Two debonding defects are in the specimen. The side lengths of the defects are, respectively, $30 \mathrm{~mm}$ and $40 \mathrm{~mm}$.

Figure 14 is the diagram of the testing principle. In testing process, the exciting probe sends an exciting wave, and, then, the receiving probe will receive excited lamb waves. The excitation wave is a square wave with a frequency of $500 \mathrm{kHz}$. The sampling frequency is $25 \mathrm{MHz}$. Three groups of signal are collected in the experiment, responding to three situations, that is, no defect, $30 \mathrm{~mm}$ defect, and $40 \mathrm{~mm}$

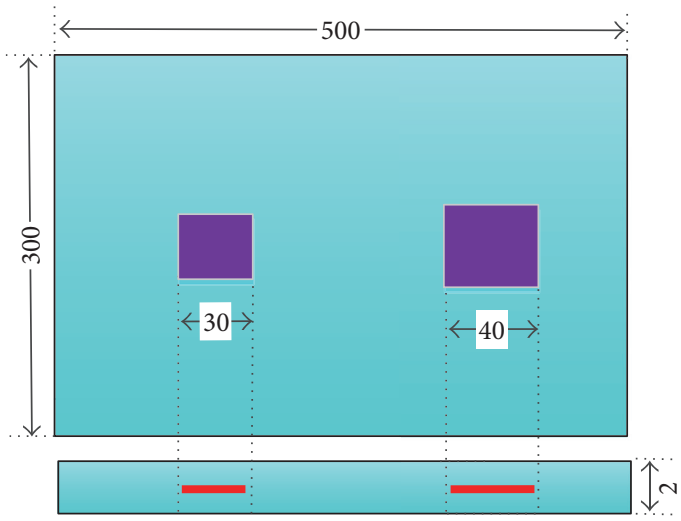

FIGURE 13: The size diagram of the specimen.

defect, respectively. The corresponding GWs collected in the experiment are presented in Figure 15.

\section{Result and Discussion}

Figures 16-18 illustrate the final IFs and IAs of three groups of experimental signals by using the presented algorithm. First, 


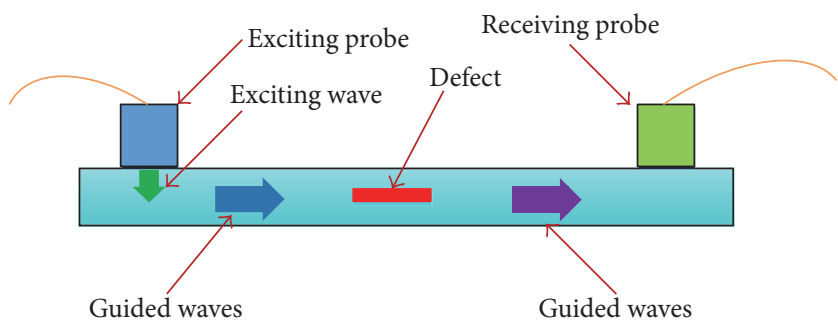

FIgURE 14: The diagram of the testing principle.

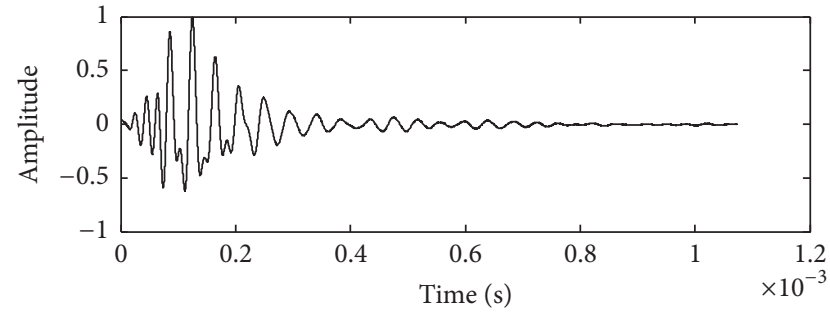

(a)

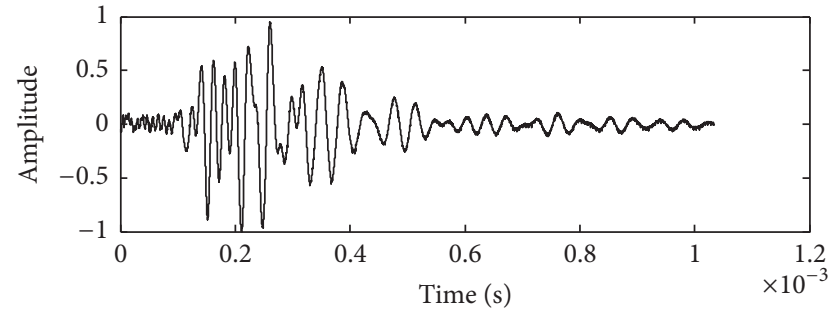

(b)

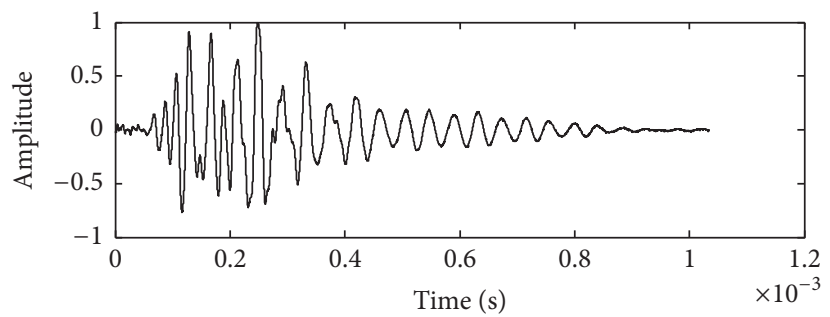

(c)

FIGURE 15: The GWs collected in the experiment: (a) no defect; (b) $30 \mathrm{~mm}$; and (c) $40 \mathrm{~mm}$.

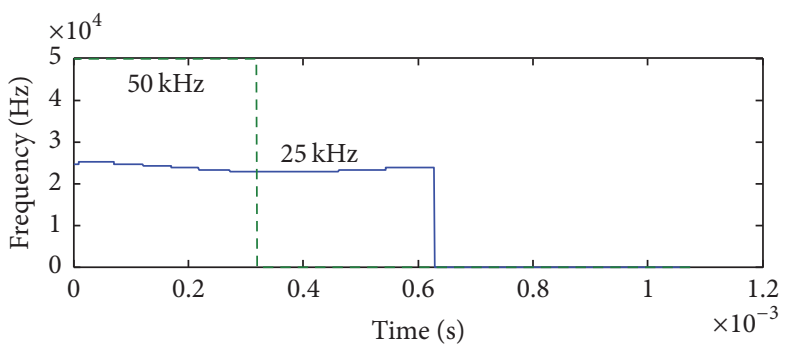

- Mode 1

- - - Mode 2

(a)

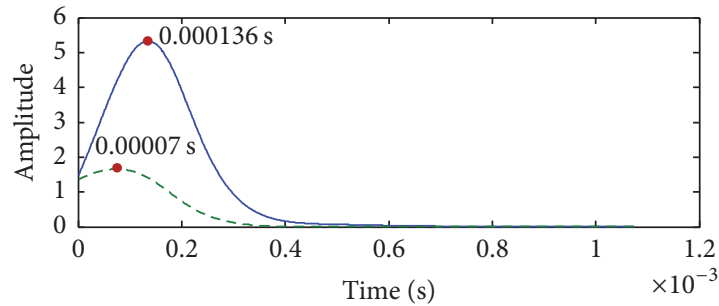

- Mode 1

(b)

FIGURE 16: The final IFs and IAs of no defect by using the presented algorithm: (a) IF and (b) IA.

two modes exist in the experimental signal of no defect and are at $50 \mathrm{kHz}$ and $25 \mathrm{kHz}$. Modes at $50.8 \mathrm{kHz}, 32.4 \mathrm{kHz}$, and $24.2 \mathrm{kHz}$ are in the signal of $30 \mathrm{~mm}$ defect. Modes at $48.9 \mathrm{kHz}$, $35.6 \mathrm{kHz}$, and $24.3 \mathrm{kHz}$ are in the signal of $40 \mathrm{~mm}$ defect. Considering that $50 \mathrm{kHz}, 50.8 \mathrm{kHz}$, and $48.9 \mathrm{kHz}$ pose little differences and the same for $25 \mathrm{kHz}, 24.2 \mathrm{kHz}$, and $24.3 \mathrm{kHz}$, it seems that defects stimulate new modes, and $32.4 \mathrm{kHz}$ corresponds to $30 \mathrm{~mm}$ defect, and $35.6 \mathrm{kHz}$ corresponds to $40 \mathrm{~mm}$ defect. On the basis of this phenomenon, we can try to detect the defect. Moreover, the frequency of the new mode becomes greater along with the defect size $(32.4 \mathrm{kHz}$ for $30 \mathrm{~mm}$, and $35.6 \mathrm{kHz}$ for $40 \mathrm{~mm}$ ). Maybe, we can do a try to evaluate the size of the defect, according to this relationship. The peak time of the mode becomes less along with the mode frequency increasing, which brings into correspondence with the conclusion that a higher frequency poses a higher propagation velocity. This phenomenon can provide a way to locate the defect. The discussions above 

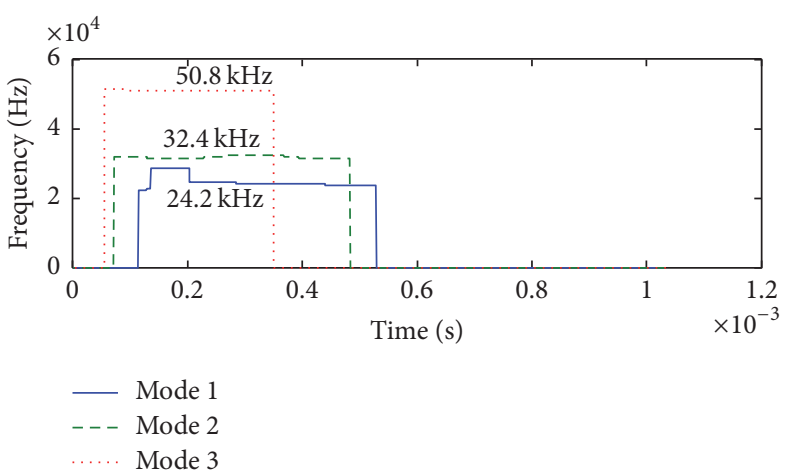

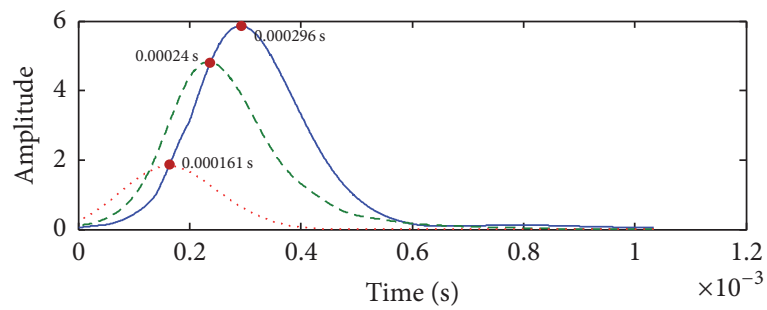

- Mode 1

- - Mode 2

Mode 3

(a)

(b)

FIGURE 17: The final IFs and IAs of $30 \mathrm{~mm}$ defect by using the presented algorithm: (a) IF and (b) IA.

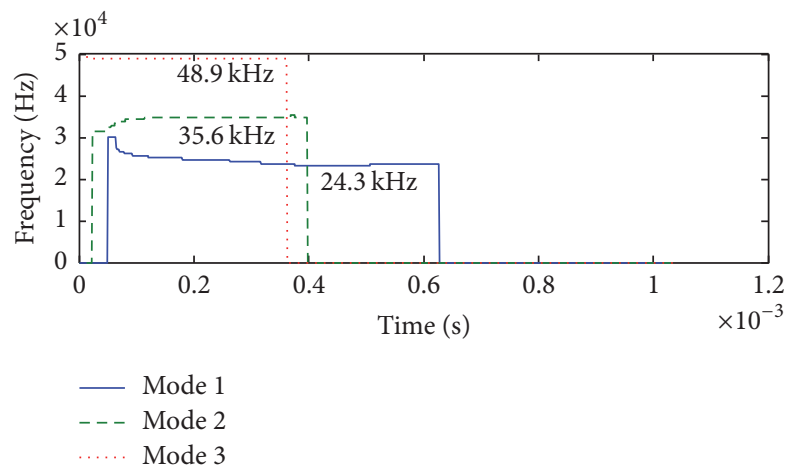

(a)

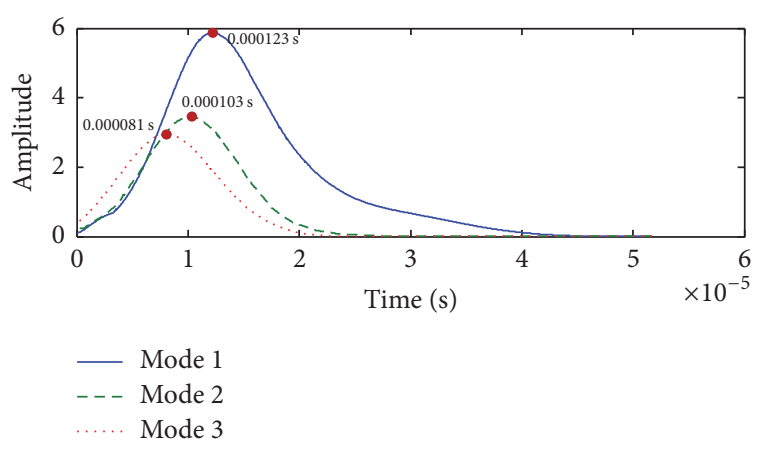

(b)

FIGURE 18: The final IFs and IAs of $40 \mathrm{~mm}$ defect by using the presented algorithm: (a) IF and (b) IA.

reveal that, on the basis of the algorithm presented in this paper, we may detect the defect, calculate the size of the defect, and locate the position of the defect.

\section{Conclusion}

This paper presents a time-frequency algorithm aiming to analyze the characteristics of ultrasonic GWs generated in a NDT for the debonding in a type of composite material. The time resolution and frequency resolution of SPWVD are promising, which is advantageous to obtain the frequencies and peak times of different GW modes. On the basis of the presented algorithm, the characteristics of the experimental signals were investigated. The result shows that new modes can be stimulated by the debonding defect, and the frequencies of new modes also change along with the size of defect. Based on the presented algorithm, we can detect the presentence, calculate size, and locate the debonding defect. The technique also can be applied in analogue NDTs and NDEs on the basis of the ultrasonic GWs. Further research will be done to validate the feasibility for locating defects by the algorithm.

\section{Competing Interests}

The authors declare that they have no competing interests.

\section{References}

[1] W. J. Cantwell and J. Morton, "The significance of damage and defects and their detection in composite materials: a review," The Journal of Strain Analysis for Engineering Design, vol. 27, no. 1, pp. 29-42, 1992.

[2] S. S. Kessler, S. M. Spearing, and C. Soutis, "Damage detection in composite materials using Lamb wave methods," Smart Materials and Structures, vol. 11, no. 2, pp. 269-278, 2002.

[3] T. Liu, W. Zhang, and S. Yan, "A novel image enhancement algorithm based on stationary wavelet transform for infrared thermography to the de-bonding defect in solid rocket motors," Mechanical Systems and Signal Processing, vol. 62, no. 10, pp. 366-380, 2015.

[4] M. P. Hentschel, K.-W. Harbich, and A. Lange, "Nondestructive evaluation of single fibre debonding in composites by X-ray refraction," NDT \& E International, vol. 27, no. 5, pp. 275-280, 1994.

[5] K. Diamanti, J. M. Hodgkinson, and C. Soutis, "Detection of low-velocity impact damage in composite plates using lamb waves," Structural Health Monitoring, vol. 3, no. 1, pp. 33-41, 2004.

[6] K. S. Tan, N. Guo, B. S. Wong, and C. G. Tui, "Experimental evaluation of delaminations in composite plates by the use of Lamb waves," Composites Science and Technology, vol. 53, no. 1, pp. 77-84, 1995. 
[7] W. H. Prosser, M. D. Seale, and B. T. Smith, "Time-frequency analysis of the dispersion of Lamb modes," Journal of the Acoustical Society of America, vol. 105, no. 5, pp. 2669-2676, 1999.

[8] S. W. Kercel, M. B. Klein, and B. Pouet, "Bayesian separation of Lamb wave signatures in laser ultrasonics," in Applications and Science of Computational Intelligence III, vol. 4055 of Proceedings of SPIE, pp. 350-361, April 2000.

[9] P. S. Mahadev, K. R. Vinu, K. Balasubramaniam et al., "Imaging of defects in composite structures using guided ultrasonics," in Proceedings of the SPIE 5062, Smart Materials, Structures, and Systems, International Society for Optics and Photonics, 2003.

[10] R. A. Osegueda, V. Kreinovich, S. Nazarian et al., Detection of Cracks at Rivet Holes in Thin Plates Using Lamb-Wave Scanning, NDE for Health Monitoring and Diagnostics. International Society for Optics and Photonics, 2003.

[11] W. Wu, G. Jiang, S. Huang, and C. J. Leo, "Vertical dynamic response of pile embedded in layered transversely isotropic soil," Mathematical Problems in Engineering, vol. 2014, Article ID 126916, 12 pages, 2014.

[12] T. Liu, S. Yan, and W. Zhang, "Time-frequency analysis of nonstationary vibration signals for deployable structures by using the constant-Q nonstationary gabor transform," Mechanical Systems and Signal Processing, vol. 75, pp. 228-244, 2016.

[13] D. Dhital and J. R. Lee, "A fully non-contact ultrasonic propagation imaging system for closed surface crack evaluation," Experimental Mechanics, vol. 52, no. 8, pp. 1111-1122, 2012.

[14] C. A. Paget, S. Grondel, K. Levin et al., "Damage assessment in composites by Lamb waves and wavelet coefficients," Smart Materials and Structures, vol. 12, no. 3, pp. 393-412, 2003.

[15] X. Chen and Z. Feng, "Application of reassigned wavelet scalogram in wind turbine planetary gearbox fault diagnosis under nonstationary conditions," Shock and Vibration, vol. 2016, Article ID 6723267, 12 pages, 2016.

[16] Y. Y. Kim and E.-H. Kim, "Effectiveness of the continuous wavelet transform in the analysis of some dispersive elastic waves," The Journal of the Acoustical Society of America, vol. 110, no. 1, pp. 86-94, 2001.

[17] M. R. Kaloop, J. W. Hu, M. A. Sayed, and J. Seong, "Structural performance assessment based on statistical and wavelet analysis of acceleration measurements of a building during an earthquake," Shock and Vibration, vol. 2016, Article ID 8902727, 13 pages, 2016.

[18] G. Rilling and P. Flandrin, "One or two frequencies? The empirical mode decomposition answers," IEEE Transactions on Signal Processing, vol. 56, no. 1, pp. 85-95, 2008.

[19] B. Boashash and P. J. Black, "An efficient real-time implementation of the wigner-ville distribution," IEEE Transactions on Acoustics, Speech, and Signal Processing, vol. 35, no. 11, pp. 16111618, 1987.

[20] P. Flandrin and B. Escudié, "An interpretation of the PseudoWigner-Ville distribution," Signal Processing, vol. 6, no. 1, pp. 2736, 1984.

[21] Z. Chen and R. C. Maher, "Semi-automatic classification of bird vocalizations using spectral peak tracks," The Journal of the Acoustical Society of America, vol. 120, no. 5, pp. 2974-2984, 2006.

[22] R. K. Potter, G. A. Kopp, and H. G. Kopp, Visible Speech, Dover, New York, NY, USA, 1966.

[23] Z. Wu and N. E. Huang, "Ensemble empirical mode decomposition: a noise-assisted data analysis method," Advances in Adaptive Data Analysis, vol. 1, no. 1, pp. 1-41, 2009. 


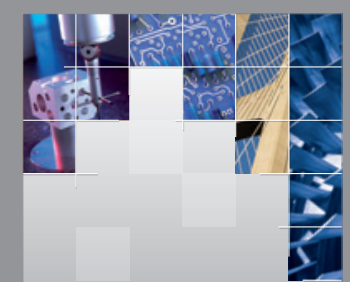

\section{Enfincering}
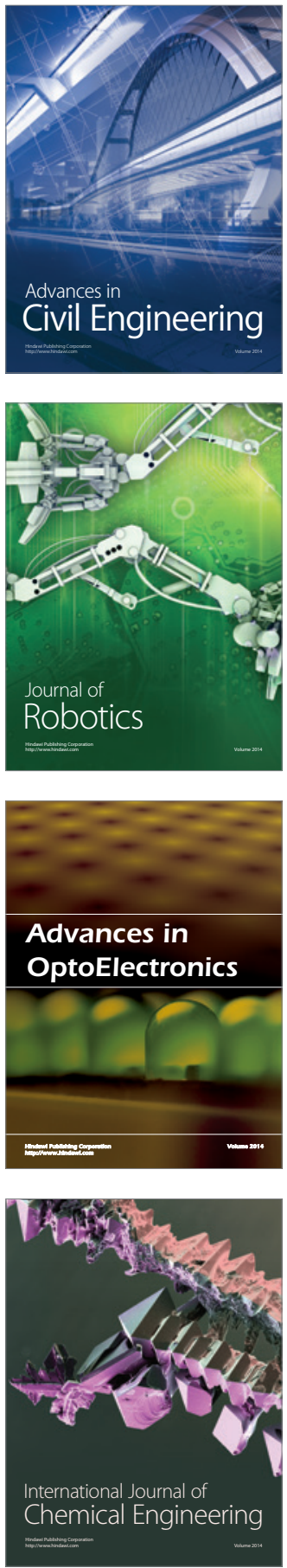

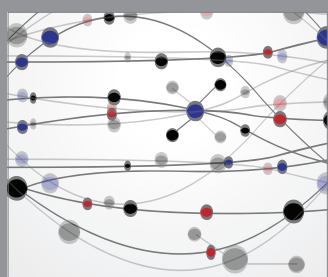

The Scientific World Journal

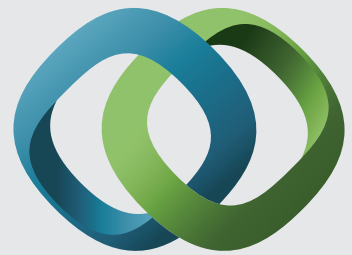

\section{Hindawi}

Submit your manuscripts at

https://www.hindawi.com
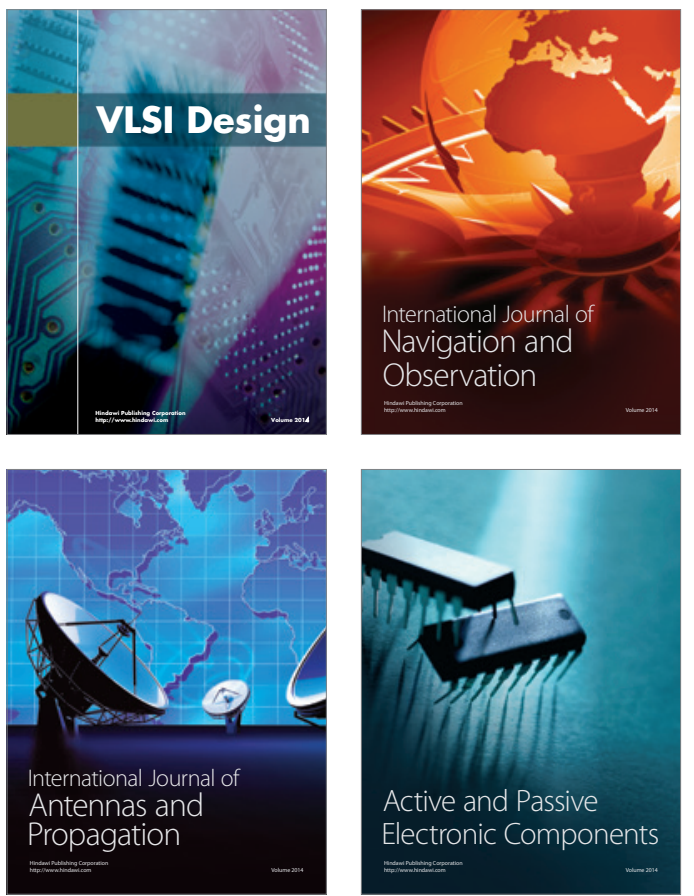
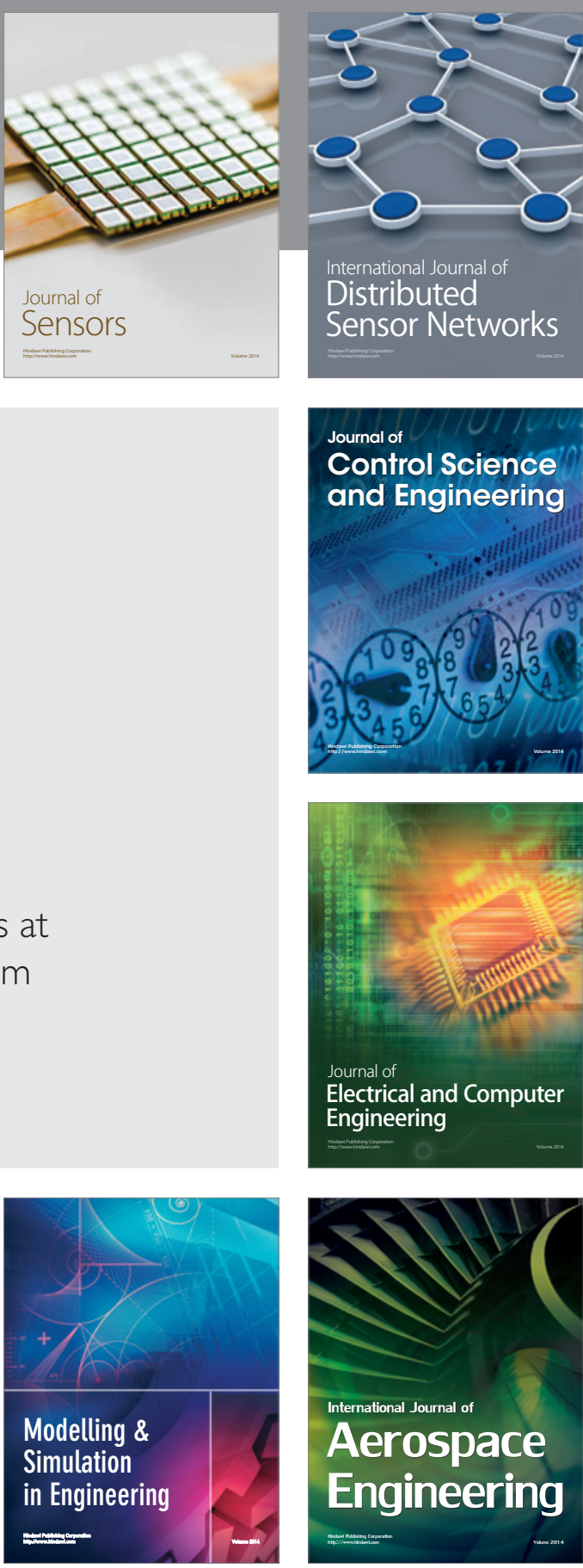

International Journal of

Distributed

Sensor Networks

$-$

Joumal of

Control Science

and Engineering
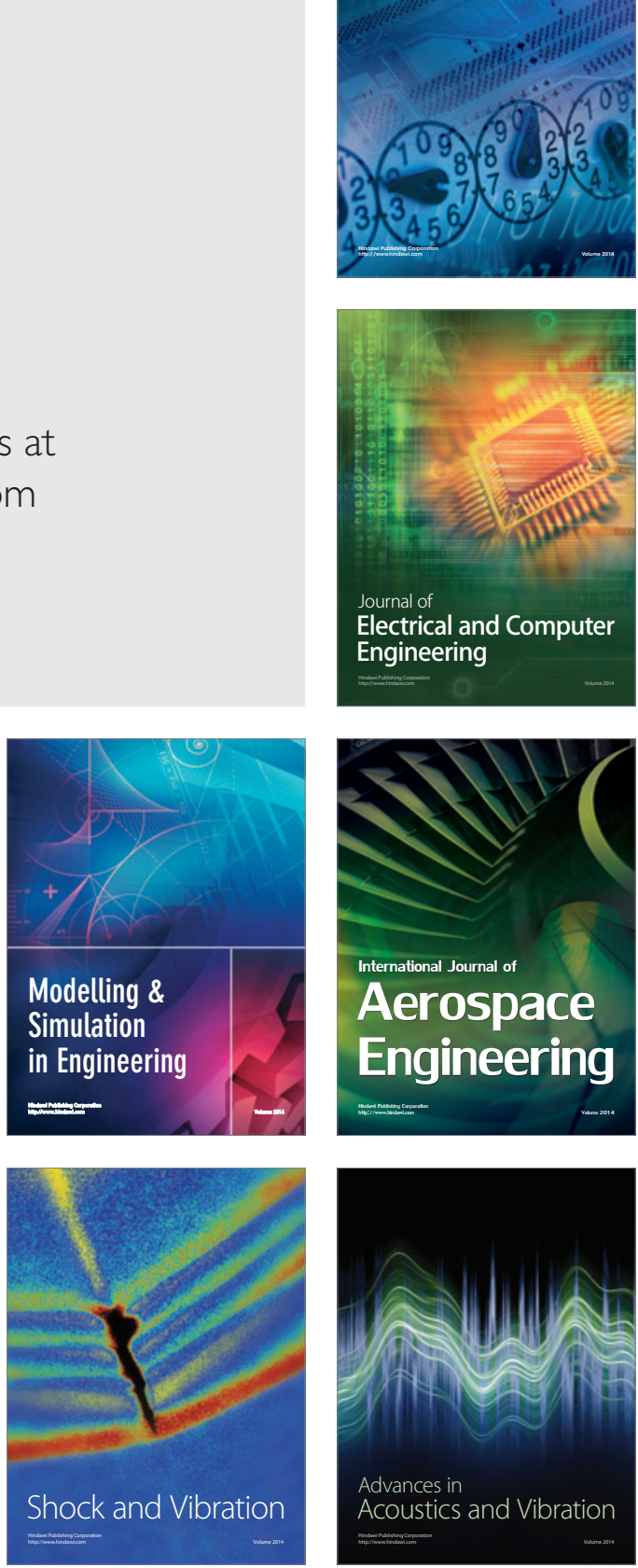